Sādhanā Vol. 30, Part 1, February 2005, pp. 1-10. @ Printed in India

\title{
Thermal effect on gravity waves in a compressible liquid layer over a solid half-space under initial hydrostatic stress
}

\author{
SUSHIL KUMAR ADDY ${ }^{1}$ and NIL RATAN CHAKRABORTY ${ }^{2}$ \\ ${ }^{1}$ Department of Mathematics and ${ }^{2}$ Department of Physics, Jamshedpur \\ Co-operative College, Jamshedpur 831 001, India \\ e-mail: nil_c53@yahoo.co.in
}

\section{MS received 12 July 2004}

\begin{abstract}
This paper deals with the effect of temperature on gravity waves in a compressible liquid layer over a solid half-space. It has been assumed that the liquid layer is under the action of gravity, while the solid half-space is under the influence of initial compressive hydrostatic stress. When the temperature of the half-space is altered, gravity waves propagate through the liquid layer along with sub-oceanic Rayleigh waves in the system. A new frequency equation has been derived here for gravity waves and sub-oceanic Rayleigh waves.

It has been shown graphically that the phase velocity of gravity waves is influenced significantly by the initial compressive hydrostatic stress present in the solid half-space, for a particular value of the phase velocity of sub-oceanic Rayleigh waves and different coupling co-efficients of the temperature.
\end{abstract}

Keywords. Thermo elasticity; liquid layer; solid half-space; initial hydrostatic stress; gravity waves; frequency equation.

\section{Introduction}

Recently the propagation of thermo-elastic waves has been discussed by many authors such as Chandrasekharia (1996) and Ahmed (2000). They have shown how the phase velocity of thermo-elastic waves is influenced by the nature of the solid medium or the initial stress present in it. This paper has discussed the effect of temperature as well as initial compressive hydrostatic stress on the propagation of gravity waves in a liquid layer over a solid halfspace and sub-oceanic Rayleigh waves in the system. It has been assumed that the liquid layer is under the action of gravity, which is over an initially stressed solid half-space. When temperature of the solid half-space is altered, gravity waves propagate through the liquid layer underlain by the solid half-space along with sub-oceanic Rayleigh waves propagating through the system. A new frequency equation of gravity waves and sub-oceanic Rayleigh waves has been derived here. The values of phase velocities of gravity waves have been computed for different values of initial compressive hydrostatic stress of the solid half-space in dimensionless form for a certain value of the phase velocity of sub-oceanic Rayleigh waves and different coupling co-efficients of the temperature. The results are plotted in graphs. The 
graphs show how the phase velocity of gravity waves alter with respect to different values of initial compressive hydrostatic stress and the coupling coefficient of the temperature.

\section{Formulation and solution of the problem}

Let us consider a compressible liquid layer of thickness $H$ over a solid half-space (figure 1). The surface of the half-space is located at $Y=0$, the $Y$-axis is directed vertically downwards and the $X$-axis is taken in the direction of wave propagation. The liquid is under the action of gravity that does not practically affect the solid half-space, unless the waves are much longer than ordinary earthquake Rayleigh waves. The half-space is under an initial compressive hydrostatic stress $S$ at an initial temperature $T_{o}$. When the temperature of the half-space is changed, incremental stresses, $s_{i j}$, together with incremental strains, $e_{i j}$, are produced in it, which are measured with reference to the rotated axes as explained by Biot (1965).

The wave equation, satisfied by the velocity potential $\bar{\phi}_{1}$ in a liquid is given by (Ewing et al 1957)

$$
\partial^{2} \bar{\phi}_{1} / \partial t^{2}=\alpha_{1}^{2} \nabla^{2} \bar{\phi}_{1}+g\left(\partial \bar{\phi}_{1} / \partial t\right)
$$

where $\alpha_{1}^{2}=\lambda_{1} / \rho_{1} ; \lambda_{1}$ being Lame's constant and $\rho_{1}$ the density of the liquid.

For plane harmonic waves propagating along the $x$-axis in the liquid layer, we take

$$
\bar{\phi}_{1}=f_{1}(y) \exp [i(\omega t-k x)] .
$$

Substituting the value of $\bar{\phi}_{1}$ into (1) we get

$$
\alpha_{1}^{2}\left(\mathrm{~d}^{2} f_{1} / \mathrm{d} y^{2}\right)+g\left(\mathrm{~d} f_{1} / \mathrm{d} y\right)-\left(k^{2} \alpha_{1}^{2}-\omega^{2}\right) f_{1}=0
$$

The solution of (3) is

$$
f_{1}(y)=e^{-g y / 2 \alpha_{1}^{2}}\left[A e^{-i \eta y}+B e^{i \eta y}\right]
$$

where

$$
\eta=\left(k_{\alpha 1}^{2}-k^{2}-\left(g^{2} / 4 \alpha_{1}^{4}\right)\right)^{1 / 2}, \text { where } k_{\alpha 1}=\omega / \alpha_{1} .
$$

From (2) and (4), we get

$$
\bar{\phi}_{1}=\exp \left[-\left(g y / 2 \alpha_{1}^{2}\right)+i(\omega t-k x)\right]\left[A e^{-i \eta y}+B e^{i \eta y}\right] .
$$

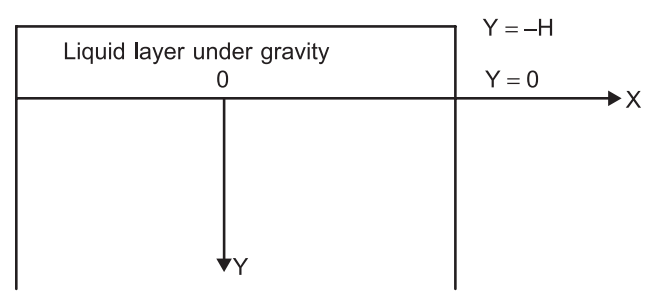

Figure 1. Solid half-space under initial hydrostatic stress. 
The velocity components in the liquid along the $X$ and $Y$-axes respectively are given by

$$
\begin{aligned}
\bar{u}_{1}= & \frac{\partial \bar{\phi}_{1}}{\partial x}=-i k \exp \left[i(\omega t-k x)-\frac{g y}{2 \alpha_{1}^{2}}\right]\left[A e^{-i \eta y}+B e^{i \eta y}\right] \\
\bar{v}_{1}= & \frac{\partial \bar{\phi}_{1}}{\partial y}=-\exp \left[i(\omega t-k x)-\frac{g y}{2 \alpha_{1}^{2}}\right]\left[\left(i \eta+\frac{g}{2 \alpha_{1}^{2}}\right) A e^{-i \eta y}\right. \\
& \left.-\left(i \eta-\frac{g}{2 \alpha_{1}^{2}}\right) B e^{i \eta y}\right]
\end{aligned}
$$

For the solid half-space, the dynamical equations of equilibrium are given by (Biot 1965)

$$
\begin{aligned}
& \left(\partial s_{11} / \partial x\right)+\left(\partial s_{12} / \partial y\right)=\rho_{2}\left(\partial^{2} u / \partial t^{2}\right), \\
& \left(\partial s_{12} / \partial x\right)+\left(\partial s_{22} / \partial y\right)=\rho_{2}\left(\partial^{2} v / \partial t^{2}\right),
\end{aligned}
$$

where $\rho_{2}$ is the density of the solid half-space.

The thermal stress-strain relations with incremental isotropy are given by (Nowacki 1975)

$$
\begin{aligned}
& s_{11}=\lambda_{2}\left(e_{x x}+e_{y y}\right)+2 \mu_{2} e_{x x}-\gamma T, \\
& s_{22}=\lambda_{2}\left(e_{x x}+e_{y y}\right)+2 \mu_{2} e_{y y}-\gamma T, \\
& s_{12}=2 \mu_{2} e_{x y},
\end{aligned}
$$

where $T$ is the incremental change of temperature from the initial state, $\gamma=\left(3 \lambda_{2}+\right.$ $\left.2 \mu_{2}\right) \alpha_{t}, \lambda_{2}, \mu_{2}$ are Lame's constants and $\alpha_{t}$ is the coefficient of linear expansion of the solid half-space.

The incremental strain components are given by (Biot 1965)

$$
\begin{aligned}
& e_{x x}=\partial u_{2} / \partial x, \\
& e_{y y}=\partial v_{2} / \partial y, \\
& e_{x y}=\frac{1}{2}\left[\left(\partial v_{2} / \partial x\right)+\left(\partial u_{2} / \partial y\right)\right],
\end{aligned}
$$

where $u_{2}$ and $v_{2}$ are the displacement components in the solid half-space along the $X$-axis and $Y$-axis respectively.

Equations (7) with the help of (8) and (9) change to

$$
\begin{aligned}
& \left(\lambda_{2}+2 \mu_{2}\right) \frac{\partial^{2} u_{2}}{\partial x^{2}}+\left(\lambda_{2}+\mu_{2}\right) \frac{\partial^{2} v_{2}}{\partial x \partial y}+\mu_{2} \frac{\partial^{2} u_{2}}{\partial y^{2}}=\rho_{2} \frac{\partial^{2} u_{2}}{\partial t^{2}}+\frac{\partial}{\partial x}(\gamma T), \\
& \left(\lambda_{2}+2 \mu_{2}\right) \frac{\partial^{2} v_{2}}{\partial y^{2}}+\left(\lambda_{2}+\mu_{2}\right) \frac{\partial^{2} u_{2}}{\partial x \partial y}+\mu_{2} \frac{\partial^{2} v_{2}}{\partial x^{2}}=\rho_{2} \frac{\partial^{2} v_{2}}{\partial t^{2}}+\frac{\partial}{\partial y}(\gamma T) .
\end{aligned}
$$

Displacement components $u_{2}$ and $v_{2}$ may be expressed in terms of the functions $\phi_{2}$ and $\psi_{2}$ as follows:

$$
\begin{aligned}
& u_{2}=\left(\partial \phi_{2} / \partial x\right)-\left(\partial \psi_{2} / \partial y\right), \\
& v_{2}=\left(\partial \phi_{2} / \partial y\right)+\left(\partial \psi_{2} / \partial x\right) .
\end{aligned}
$$


Equations (10) and (11) show that the functions $\phi_{2}$ and $\psi_{2}$ satisfy the wave equations:

$$
\begin{aligned}
\nabla^{2} \phi_{2} & =\frac{\rho_{2}}{\lambda_{2}+2 \mu_{2}} \frac{\partial^{2} \phi_{2}}{\partial t^{2}}+\frac{\gamma T}{\lambda_{2}+2 \mu_{2}}, \\
\nabla^{2} \psi_{2} & =\frac{\rho_{2}}{\mu_{2}} \frac{\partial^{2} \psi_{2}}{\partial t^{2}}
\end{aligned}
$$

where $\nabla^{2}=\left(\partial^{2} / \partial x^{2}\right)+\left(\partial^{2} / \partial y^{2}\right)$.

The heat conduction equation in the solid half-space is given by (Nowacki 1975)

$$
\nabla^{2} T-\frac{s \rho_{2}}{\delta} \frac{\partial T}{\partial t}-\frac{\gamma T_{0}}{\delta} \nabla^{2}\left(\frac{\partial \phi_{2}}{\partial t}\right)=0
$$

where $s$ is the specific heat capacity and $\delta$ is the thermal conductivity of the solid half-space.

Eliminating $T$ from (12a) and (13) and retaining (12b), we get

$$
\left(\nabla^{2}-\frac{1}{C_{1}^{2}} \frac{\partial^{2}}{\partial t^{2}}\right)\left(\nabla^{2}-\frac{s \rho_{2}}{\delta} \frac{\partial}{\partial t}\right) \phi_{2}-\frac{\gamma^{2} T_{0}}{\left(\lambda_{2}+2 \mu_{2}\right) \delta} \nabla^{2}\left(\frac{\partial \phi_{2}}{\partial t}\right)=0
$$

and

$$
\left(\nabla^{2}-\frac{1}{C_{2}^{2}} \frac{\partial^{2}}{\partial t^{2}}\right) \psi_{2}=0
$$

where

$$
C_{1}^{2}=\frac{\lambda_{2}+2 \mu_{2}}{\rho_{2}} \quad \text { and } \quad C_{2}^{2}=\frac{\mu_{2}}{\rho_{2}} .
$$

The solutions of (14) are chosen in the form:

$$
\begin{aligned}
& \phi_{2}(x, y, t)=f_{2}(y) \exp [i(\omega t-k x)], \\
& \psi_{2}(x, y, t)=g_{2}(y) \exp [i(\omega t-k x)] .
\end{aligned}
$$

Putting these values of $\phi_{2}$ and $\psi_{2}$ in (14), we get the following differential equations:

$$
\begin{aligned}
& \left(\frac{\partial^{2}}{\partial y^{2}}-\delta_{1}^{2}\right)\left(\frac{\partial^{2}}{\partial y^{2}}-\delta_{2}^{2}\right) f_{2}(y)=0, \\
& \left(\frac{\partial^{2}}{\partial y^{2}}-v^{2}\right) g_{2}(y)=0,
\end{aligned}
$$

where $\delta_{1}^{2}=k^{2}-k_{1}^{2}, \delta_{2}^{2}=k^{2}-k_{2}^{2}, v^{2}=k^{2}-\Gamma^{2}$ and $\Gamma^{2}=\omega^{2} / C_{2}^{2}$.

Here $k_{1}^{2}$ and $k_{2}^{2}$ are the roots of the biquadratic equation.

$$
Z^{4}-Z^{2}\left[\sigma^{2}+q(1+\epsilon)\right]+\sigma^{2} q=0, \quad \text { where } Z^{2}=-\nabla^{2},
$$

which are given by $k_{1}^{2}=q\left[1+\frac{q \epsilon}{q-\sigma^{2}}\right]$ and $k_{2}^{2}=\sigma^{2}\left[1-\frac{q \epsilon}{q-\sigma^{2}}\right]$. 
Also $\sigma^{2}=\omega^{2} / C_{1}^{2}, q=-i \omega s \rho_{2} / \delta$ and $\epsilon=\gamma^{2} T_{0} /\left[s \rho_{2}\left(\lambda_{2}+2 \mu_{2}\right)\right]$.

The stresses and hence the functions $\phi_{2}$ and $\psi_{2}$ vanish as $x^{2}+y^{2}$ tends to infinity. Hence we get the following solutions of (17):

$$
\begin{aligned}
& f_{2}(y)=(C / i \omega) e^{-\delta_{1} y}+(D / i \omega) e^{-\delta_{2} y}, \\
& g_{2}(y)=(E / i \omega) e^{-v y} .
\end{aligned}
$$

Combining (16) and (19), we get

$$
\begin{aligned}
& \phi_{2}(x, y, t)=(1 / i \omega)\left[C e^{-\delta_{1} y}+D e^{-\delta_{2} y}\right] \exp [i(\omega t-k x)], \\
& \psi_{2}(x, y, t)=(E / i \omega) e^{-v y} \exp [i(\omega t-k x)] .
\end{aligned}
$$

Equations (11) and (20) together give

$$
\begin{aligned}
& u_{2}=(1 / i \omega)\left[-i k\left(C e^{-\delta_{1} y}+D e^{-\delta_{2} y}\right)+v E e^{-v y}\right] \exp [i(\omega t-k x)], \\
& v_{2}=-(1 / i \omega)\left[i k E e^{-v y}+\delta_{1} C e^{-\delta_{1} y}+\delta_{2} D e^{-\delta_{2} y}\right] \exp [i(\omega t-k x)] .
\end{aligned}
$$

Velocity components in the solid half-space are given by

$$
\begin{aligned}
& \bar{u}_{2}=\partial u_{2} / \partial t=-\left[i k\left(C e^{-\delta_{1} y}+D e^{-\delta_{2} y}\right)-v E e^{-v y}\right] \exp [i(\omega t-k x)], \\
& \bar{v}_{2}=\partial v_{2} / \partial t=-\left[\delta_{1} C e^{-\delta_{1} y}+\delta_{2} D e^{-\delta_{2} y}+i K E e^{-v y}\right] \exp [i(\omega t-k x)] .
\end{aligned}
$$

$T$ is given by

$$
T=\frac{\lambda_{2}+2 \mu_{2}}{\gamma}\left[\nabla^{2}-\frac{1}{C_{1}^{2}} \frac{\partial^{2}}{\partial t^{2}}\right] \phi_{2} .
$$

Combining the first equations of (20) with (23), we get

$$
\left.T=\frac{\lambda_{2}+2 \mu_{2}}{\gamma i \omega}\left[\eta_{1} C e^{-\delta_{1} y}+\eta_{2} D e^{-\delta_{2} y}\right)\right] \exp [i(\omega t-k x)]
$$

where $\eta_{1}=\sigma^{2}-k_{1}^{2}$ and $\eta_{2}=\sigma^{2}-k_{2}^{2}$.

\section{Boundary conditions}

Boundary conditions require that pressure is zero at the free surface of the liquid, whereas the vertical velocity component together with the tangential and normal forces per unit area is continuous at the surface $Y=0$. These conditions are equivalent to

$$
\begin{aligned}
p & =0, \text { at } Y=-H, \\
\bar{v}_{1} & =\bar{v}_{2}, \text { at } Y=0, \\
\Delta f_{x} & =s_{12}+S\left(\partial v_{2} / \partial x\right)=0, \text { at } Y=0, \\
p & =-\Delta f_{y},
\end{aligned}
$$


where

$$
\begin{aligned}
\Delta f_{y} & =s_{22}-S\left(\partial u_{2} / \partial x\right)-g \rho_{2}\left(v_{2}\right)_{y=0}, \\
(\partial T / \partial y)+h T & =0, \text { at } Y=0,
\end{aligned}
$$

where $\Delta f_{x}$ and $\Delta f_{y}$ are the incremental boundary forces per unit initial area and $h$ is the ratio of the co-efficient of heat transfer and thermal conductivity.

Considering the deformation of the free surface and denoting the vertical displacement by $v_{1}$, the first boundary condition (25a) becomes

$$
-\left(\partial \bar{\phi}_{1} / \partial t\right)+g v_{1}=0 .
$$

Using (5), (6) and $\bar{v}_{1}=i \omega v_{1}$ at $y=-H,(26)$ becomes

$$
A\left(-\omega^{2}+i \eta g+\frac{g^{2}}{2 \alpha_{1}^{2}}\right) e^{i \eta H}+B\left(-\omega^{2}-i \eta g+\frac{g^{2}}{2 \alpha_{1}^{2}}\right) e^{-i \eta H}=0 .
$$

Using (6) and (22), the second boundary condition (25b) becomes

$$
-\left(i \eta+\frac{g}{2 \alpha_{1}^{2}}\right) A+\left(i \eta-\frac{g}{2 \alpha_{1}^{2}}\right) B+\delta_{1} C+\delta_{2} D+i k E=0 .
$$

Using (8), (9), (11) and (21), the third boundary condition (25c) becomes

$$
(1+\zeta) i k \delta_{1} C+(1+\zeta) i k \delta_{2} D+\left[\left(\Gamma^{2} / 2\right)-(1+\zeta) k^{2}\right] E=0,
$$

where $\zeta=S / 2 \mu$.

Now the tangential force per unit area on the side of the liquid is given by (Ewing et al 1957)

$$
p=-\rho_{1}\left(\partial \bar{\phi}_{1} / \partial t\right)+\rho_{1} g\left(v_{1}\right)_{y=0},
$$

i.e

$$
\begin{aligned}
p= & {\left[-\rho_{1}\left(i \omega+\frac{\eta g}{\omega}+\frac{g^{2}}{i \omega 2 \alpha_{1}^{2}}\right) A-\rho_{1}\left(i \omega-\frac{\eta g}{\omega}+\frac{g^{2}}{i \omega 2 \alpha_{1}^{2}}\right) B\right] } \\
& \times \exp i(\omega t-k x) .
\end{aligned}
$$

Using (8), (9) and (21), the fourth boundary condition (25d) becomes

$$
\begin{gathered}
\rho_{1}\left[i \omega+\frac{g}{\omega} \eta+\frac{g^{2}}{i \omega 2 \alpha_{1}^{2}}\right] A+\rho_{1}\left[i \omega-\frac{g}{\omega} \eta+\frac{g^{2}}{i \omega 2 \alpha_{1}^{2}}\right] B \\
-\rho_{2}\left[i \omega\left(1-\frac{2 k^{2}}{\Gamma^{2}}\right)-\frac{i g \delta_{1}}{\omega}-\frac{i S k^{2}}{\rho_{2} \omega}\right] C \\
-\rho_{2}\left[i \omega\left(1-\frac{2 k^{2}}{\Gamma^{2}}\right)-\frac{i g \delta_{2}}{\omega}-\frac{i S k^{2}}{\rho_{2} \omega}\right] D \\
-\rho_{2}\left[\frac{2 k \omega v}{\Gamma^{2}}+\frac{g k}{\omega}+\frac{k v S}{\omega \rho_{2}}\right] E=0
\end{gathered}
$$


Using (24), the fifth boundary condition (25e) becomes

$$
\left(h-\delta_{1}\right) \eta_{1} C+\left(h-\delta_{2}\right) \eta_{2} D=0
$$

Thus the boundary conditions yield a system of five homogeneous equations (27)-(31). Eliminating the constants A, B, C, D and E from these equations, we get a fifth-order vanishing determinant. Expanding the determinant, we have the following frequency equation:

$$
\begin{aligned}
& {\left[1-\frac{g K^{2}}{\omega^{2}} \frac{\tan \eta H}{\eta}+\frac{g}{2 \alpha_{1}^{2}} \frac{\tan \eta H}{\eta}\right]\left[\left\{\left(\frac{2 k^{2}}{\Gamma^{2}}-1\right)^{2}+2 \frac{2 k^{2}}{\Gamma^{2}}\left(\frac{2 k^{2}}{\Gamma^{2}}-1\right) \zeta\right.\right.} \\
& \left.\quad+4 \frac{k^{4}}{\Gamma^{4}} \zeta^{2}\right\} \times\left\{h\left(\eta_{2}-\eta_{1}\right)+\left(\delta_{1} \eta_{1}-\delta_{2} \eta_{2}\right)\right\} \\
& \left.\quad-\left\{\frac{g}{\omega^{2}}+\frac{4 k^{2}}{\Gamma^{4}}\left(k^{2}-\Gamma^{2}\right)^{1 / 2}(1+\zeta)^{2}\right\} \times\left\{h\left(\delta_{1} \eta_{2}-\delta_{2} \eta_{1}\right)+\delta_{1} \delta_{2}\left(\eta_{1}-\eta_{2}\right)\right\}\right] \\
& \quad+\frac{\rho_{1}}{\rho_{2}} \frac{\tan \eta H}{\eta}\left[1-\left(\frac{g K}{\omega^{2}}\right)^{2}\right]\left[h\left(\delta_{1} \eta_{2}-\delta_{2} \eta_{1}\right)+\delta_{1} \delta_{2}\left(\eta_{1}-\eta_{2}\right)\right]=0
\end{aligned}
$$

where we use $v=\left(k^{2}-\Gamma^{2}\right)^{1 / 2}$.

Since $g / k_{\alpha 1}^{2} \ll 1$ for naturally occurring waves, (32) reduces to

$$
\begin{aligned}
& {\left[1-\frac{g K^{2}}{\omega^{2}} \frac{\tan \eta^{\prime} H}{\eta^{\prime}}\right]\left[\left\{\left(\frac{2 k^{2}}{\Gamma^{2}}-1\right)^{2}+2 \frac{2 k^{2}}{\Gamma^{2}}\left(\frac{2 k^{2}}{\Gamma^{2}}-1\right) \zeta+4 \frac{k^{4}}{\Gamma^{4}} \zeta^{2}\right\}\right.} \\
& \times\left\{h\left(\eta_{2}-\eta_{1}\right)+\left(\delta_{1} \eta_{1}-\delta_{2} \eta_{2}\right)\right\}-\left\{\frac{g}{\omega^{2}}+\frac{4 k^{2}}{\Gamma^{4}}\left(k^{2}-\Gamma^{2}\right)^{1 / 2}(1+\zeta)^{2}\right\} \\
& \left.\times\left\{h\left(\delta_{1} \eta_{2}-\delta_{2} \eta_{1}\right)+\delta_{1} \delta_{2}\left(\eta_{1}-\eta_{2}\right)\right\}\right]+\frac{\rho_{1}}{\rho_{2}} \frac{\tan \eta^{\prime} H}{\eta^{\prime}}\left[1-\left(\frac{g k}{\omega^{2}}\right)^{2}\right] \\
& \times\left[h\left(\delta_{1} \eta_{2}-\delta_{2} \eta_{1}\right)+\delta_{1} \delta_{2}\left(\eta_{1}-\eta_{2}\right)\right]=0,
\end{aligned}
$$

where $\eta^{\prime}=\left(k_{\alpha 1}^{2}-k^{2}\right)^{1 / 2}$.

Let

$$
\beta_{1}^{2}=1-\left(k_{1}^{2} / k^{2}\right), \quad \beta_{2}^{2}=1-\left(k_{2}^{2} / k^{2}\right) \text { and } \beta_{3}^{2}=1-\left(\Gamma^{2} / k^{2}\right)
$$

Expressing $\delta_{1}, \delta_{2}, \eta_{1}$ and $\eta_{2}$ in terms of $\beta_{1}, \beta_{2}$ and $\beta_{3}$ and using the approximation

$$
\left(k \tan \eta^{\prime} H\right) / \eta^{\prime} \rightarrow 1 \quad \text { as } \quad H \rightarrow \infty,
$$


we find that (33) reduces to the following form:

$$
\begin{gathered}
\left\{2(1+\zeta)-\frac{C^{2}}{C_{2}^{2}}\right\}^{2}\left(\frac{C^{2}}{C_{1}^{2}}-1+\beta_{1}^{2}+\beta_{2}^{2}+\beta_{1} \beta_{2}\right)-4(1+\zeta)^{2} \beta_{1} \beta_{2} \beta_{3}\left(\beta_{1}+\beta_{2}\right) \\
+\frac{\rho_{1}}{\rho_{2}} \frac{C^{4}}{C_{2}^{4}} \beta_{1} \beta_{2}\left(\beta_{1}+\beta_{2}\right)-\left(1-\frac{\rho_{1}}{\rho_{2}}\right) \frac{C^{4}}{C_{2}^{4}} \frac{g k}{\omega^{2}} \beta_{1} \beta_{2}\left(\beta_{1}+\beta_{2}\right) \\
=\frac{h}{k}\left[\left\{2(1+\zeta)-\frac{C^{2}}{C_{2}^{2}}\right\}^{2}\left(\beta_{1}+\beta_{2}\right)-4(1+\zeta)^{2} \beta_{3}\left(1+\beta_{1} \beta_{2}-\frac{C^{2}}{C_{1}^{2}}\right)\right. \\
\left.+\frac{\rho_{1}}{\rho_{2}} \frac{C^{4}}{C_{2}^{4}}\left(1+\beta_{1} \beta_{2}-\frac{C^{2}}{C_{1}^{2}}\right)-\left(1-\frac{\rho_{1}}{\rho_{2}}\right) \frac{g k}{\omega^{2}} \frac{C^{4}}{C_{2}^{4}}\left(1+\beta_{1} \beta_{2}-\frac{C^{2}}{C_{1}^{2}}\right)\right]
\end{gathered}
$$

From (18), we get

$$
k_{1}^{2}+k_{2}^{2}=\sigma^{2}+q(1+\epsilon),
$$

and

$$
k_{1}^{2} k_{2}^{2}=\sigma^{2} q .
$$

In view of (34) and (36), we have

$$
\beta_{1}^{2}+\beta_{2}^{2}=2-\left(C^{2} / C_{1}^{2}\right)-\left(i C^{2} / f C_{1}^{2}\right)(1+\epsilon)
$$

and

$$
\beta_{1}^{2} \beta_{2}^{2}=1-\left(C^{2} / C_{1}^{2}\right)-\left(i C^{2} / f C_{1}^{2}\right)\left(1+\in-\left(C^{2} / C_{1}^{2}\right)\right)
$$

where $f=\delta \omega / s \rho C_{1}^{2}$ is the reduced frequency. Substituting (37) into (35), expanding the quantities $\beta_{1}$ and $\beta_{2}$ in a series of $f$ and neglecting the terms of the order of $f^{1 / 2}$, we get a complex frequency equation, the real parts of which yield

$$
\begin{aligned}
& \left\{2(1+\zeta)-\frac{C^{2}}{C_{2}^{2}}\right\}^{2}-4(1+\zeta)^{2}\left[\left(1-\frac{C^{2}}{C_{2}^{2}}\right)\left\{1-\frac{C^{2}}{(1+\epsilon) C_{1}^{2}}\right\}\right]^{1 / 2} \\
& =\left\{\left(1-\frac{\rho_{1}}{\rho_{2}}\right) \frac{g k}{\omega^{2}}-\frac{\rho_{1}}{\rho_{2}}\right\} \frac{C^{4}}{C_{2}^{4}}\left\{1-\frac{C^{2}}{(1+\epsilon) C_{1}^{2}}\right\}^{1 / 2} .
\end{aligned}
$$

Taking $C_{2}^{2} /(1+\in) C_{1}^{2}=\theta$, (39) reduces to

$$
\begin{gathered}
\left\{2(1+\zeta)-\frac{C^{2}}{C_{2}^{2}}\right\}^{2}-4(1+\zeta)^{2}\left(1-\frac{C^{2}}{C_{2}^{2}}\right)^{1 / 2}\left(1-\frac{C^{2}}{C_{2}^{2}} \theta\right)^{1 / 2} \\
=\frac{C^{4}}{C_{2}^{4}}\left\{\left(1-\frac{\rho_{1}}{\rho_{2}}\right) \frac{g k}{\omega^{2}}-\frac{\rho_{1}}{\rho_{2}}\right\}\left(1-\frac{C^{2}}{C_{2}^{2}} \theta\right)^{1 / 2}
\end{gathered}
$$



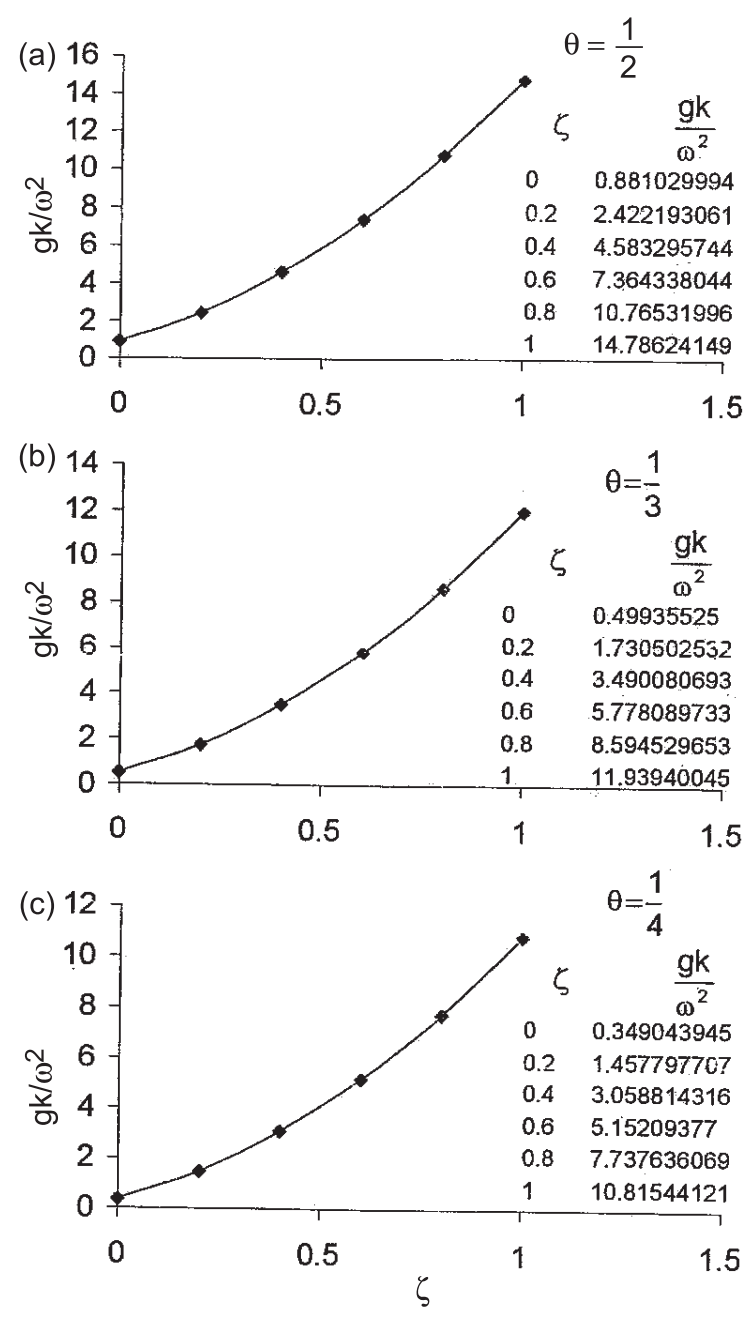

Figure 2. Graphs showing the variation of velocity of gravity waves (in dimensionless form) with initial stress.

Here $C^{2} / C_{2}^{2}$ represents the phase velocity of Rayleigh waves in the system formed by a liquid layer underlain by a solid half-space (sub-oceanic Rayleigh waves), whereas $g k / \omega^{2}$ represents the phase velocity of gravity waves, both in dimensionless form. The separation into two types of propagation arises from the great disparity in phase velocities of Rayleigh waves and gravity waves.

We have assumed that $\rho_{1} / \rho_{2}=1 / 3$, and $C^{2} / C_{2}^{2}=0.8453$. For different values of $\zeta$ and $\theta$, corresponding values of $g k / \omega^{2}$ are calculated and these are plotted in graphs shown in figure 2 .

The graphs show that as the initial compressive hydrostatic stress increases, the phase velocity of gravity waves increases in nonlinear form. They also show that when the value of the coupling coefficient of the temperature increases, then the phase velocity of gravity waves also increases, provided the initial compressive hydrostatic stress is not altered. 


\section{References}

Ahmed S M 2000 Rayleigh waves in thermo-elastic granular medium under initial stress. J. Math. Math. Sci. 23: 627-637

Biot M A 1965 Mechanics of incremental deformations (New York: John Wiley and Sons)

Chandrasekharia D S 1996 One-dimensional wave propagation in the linear theory of thermo-elasticity without energy dissipation. J. Thermal Stresses 19: 695-710

Ewing W M, Jardetzky W S, Press F 1957 Elastic waves in layered media (New York: Mc-Graw Hill)

Nowacki W 1975 Dynamic problems of thermo-elasticity (Leyden: Noordhoff) 